\title{
Tocotrienols and breast cancer: the evidence to date
}

\author{
Kalanithi Nesaretnam - Puvaneswari Meganathan • \\ Sheela Devi Veerasenan · Kanga Rani Selvaduray
}

Received: 24 March 2011/Accepted: 5 April 2011/Published online: 24 April 2011

(c) Springer-Verlag 2011

\begin{abstract}
Breast cancer is the second most frequent cancer affecting women worldwide after lung cancer. The toxicity factor associated with synthetic drugs has turned the attention toward natural compounds as the primary focus of interest as anticancer agents. Vitamin E derivatives consisting of the well-established tocopherols and their analogs namely tocotrienols have been extensively studied due to their remarkable biological properties. While tocopherols have failed to offer protection, tocotrienols, in particular, $\alpha-, \delta$-, and $\gamma$-tocotrienols alone and in combination have demonstrated anticancer properties. The discovery of the antiangiogenic, antiproliferative, and apoptotic effects of tocotrienols, as well as their role as an inducer of immunological functions, not only reveals a new horizon as a potent antitumor agent but also reinforces the notion that tocotrienols are indeed more than antioxidants. On the basis of a transcriptomic platform, we have recently demonstrated a novel mechanism for tocotrienol activity that involves estrogen receptor (ER) signaling. In silico simulations and in vitro binding analyses indicate a high affinity of specific forms of tocotrienols for $\operatorname{ER} \beta$, but not for $\mathrm{ER} \alpha$. Moreover, we have demonstrated that specific tocotrienols increase $\operatorname{ER} \beta$ translocation into the nucleus which, in turn, activates the expression of estrogenresponsive genes (MIC-1, EGR-1 and Cathepsin D) in
\end{abstract}

This paper is part of a Special Issue entitled "Tocotrienols in health and disease" (Guest editors: Bharat B. Aggarwal and Kalaniti Nesaretnam).

K. Nesaretnam $(\bowtie) \cdot$ P. Meganathan · S. D. Veerasenan ·

K. R. Selvaduray

Product Development and Advisory Services Division,

Malaysian Palm Oil Board, No.6 Persiaran Institusi,

Bandar Baru Bangi, 43000 Kajang, Selangor, Malaysia

e-mail: sarnesar@mpob.gov.my breast cancer cells only expressing ER $\beta$ cells (MDA-MB231) and in cells expressing both ER isoforms (MCF-7). The binding of specific tocotrienol forms to ER $\beta$ is associated with the alteration of cell morphology, caspase-3 activation, DNA fragmentation, and apoptosis. Furthermore, a recently concluded clinical trial seems to suggest that tocotrienols in combination with tamoxifen may have the potential to extend breast cancer-specific survival.

Keywords Tocotrienols - Anticancer - Antiproliferative · Tamoxifen $\cdot$ Apoptosis $\cdot$ Chemopreventive

\section{Introduction}

Cancer is a leading cause of death worldwide. The disease accounts for 1.38 million new cases diagnosed in 2008 worldwide (Charts Bin 2010). In the United States of America, about 1 in 8 women will develop invasive breast cancer in her lifetime (Org 2010) with 207,900 new cases and 39,840 deaths estimated in 2010 (National Cancer Institute 2010; Cancer.Org 2010). Major risk factors for breast cancer include a family history of breast cancer, early menarche, late age at first child birth, late age at menopause, and history of benign breast disease. With the exception of the genetic predisposition to the disease, the rest of the risk factors point to the lifetime exposure of women to estrogen. Estrogen does not cause the disease but is involved in the progression and development of breast cancer (Platet et al. 2004). Antiestrogens are therefore used as therapy in the control of breast cancer progression (Ingram et al. 1991).

Tamoxifen has been the standard first-line endocrine therapy for patients with estrogen receptor (ER)-positive tumors. However, almost all patients with advanced 
disease develop tamoxifen resistance. This has lead to a search for new potent antiestrogens (Fog et al. 2005; Biswas et al. 2001).

International variation in breast cancer incidence rates and changes in incidence among migrant population indicate that breast cancer risk is also influenced by environmental factors, in particular diet, and is therefore preventable. Recent scientific investigations have been made to discover possible functional properties of antioxidants in the diet, which could be efficient in preventing diseases like cancer. One such antioxidant is tocotrienols. Tocotrienols are isomers of vitamin $\mathrm{E}$ and are found in abundance in palm oil (Sylvester and Shah 2005).

Various tocotrienol isomers of vitamin $\mathrm{E}$ in palm oil have been reported to have interesting biological and physiological properties. They include potential blood cholesterol-lowering and cardioprotective effects, more efficient antioxidant activity in biological systems, and possible anticancer and neuroprotective effects (Nesaretnam 2008).

This review will summarize the evidence to date on breast cancer and tocotrienols.

\section{Historical perspective of palm tocotrienols}

Initial studies have shown that unlike many other fats and oils, palm oil does not enhance the yield of chemically induced mammary tumors when fed to rats at high levels (20\% w/w fat) in a semi-purified diet (Sylvester et al. 1986; Sundram et al. 1989). It was then reported that linoleic acid which was known to promote mammary carcinogenesis was present in only minute amounts in palm oil. This may have accounted for the lower tumor-promoting effect of palm oil compared with the other fats tested (Dayton et al. 1977). Subsequently, however, Nesaretnam et al. (1992), reported that palm oil stripped of vitamin E-promoted tumorigenesis in rats induced with DMBA. This was the first study to provide the evidence that vitamin $\mathrm{E}$ mainly consisting of tocotrienols and tocopherols contributed to the anticarcinogenic effect of palm oil. Further studies in vitro showed that only tocotrienols had anticarcinogenic properties while tocopherols failed to show their anticarcinogenic properties.

\section{In vitro studies}

In the last decade, tocotrienols have gained much attention due to their antioxidant and antiproliferative nature in many human tumors (Nesaretnam et al. 2007). An in vitro study by Nesaretnam et al. (1995), revealed that the tocotrienol-rich fraction or TRF was able to inhibit the MDA-
MB-435 cell proliferation by $50 \%$ at only $180 \mu \mathrm{g} / \mathrm{mL}$. Interestingly, a subsequent study on ZR-75-1 breast cancer cells only required $6 \mu \mathrm{g} / \mathrm{mL}$ of tocotrienols to exert growth-inhibitory effects (Nesaretnam et al. 2000).

In the prognosis of breast cancer, the status of estrogen receptor (ER) is used. Prior to 1996, only ER- $\alpha$ existed. In 1996, however, a second estrogen receptor was discovered and named as ER- $\beta$. This receptor was found to be more expressed compared with ER- $\alpha$ in cancer cells. Both receptors exert opposite effects in the activator protein 1 (AP-1) signaling pathway. ER- $\alpha$ activates transcription while ER- $\beta$ inhibits it (Balfe et al. 2004). Two ER- $\beta$ cohorts exist; one is coexpressed with ER- $\alpha$, and the other is not. The latter has been investigated as a potential target for anticancer mechanisms as well as therapy (Cullen et al. 2001; Skliris et al. 2008). A recent study by Comitato et al., revealed a novel mechanism for tocotrienols targeting ER signaling. High-affinity binding existed between tocotrienols and ER- $\beta$ through hydrogen bonding as demonstrated by in silico simulations and in vitro binding analysis in MDA-MB-231 cell lines. This interaction enhanced the ER- $\beta$ translocation into the nucleus to activate estrogenresponsive genes (MIC-1, EGP-1, and cathepsin D). Overexpression of MIC-1 by tocotrienol induced cell cycle arrest in S-phase and apoptosis. Alteration of cell morphology, DNA fragmentation, and caspase-3 activation associated with tocotrienol treatment has been suggested as the molecular mechanism contributing to the effectiveness of $\gamma$ - and $\delta$-tocotrienols (Comitato et al. 2009). Following this observation, Comitato and colleagues conducted a further study on MCF-7 cells that express both ER- $\alpha$ and ER- $\beta$. Significant inhibition of ER- $\alpha$ was observed following elevated levels of ER- $\beta$ nuclear translocation implying a novel mechanism of action for tocotrienols (Comitato et al. 2010).

The effects of TRF and $\alpha$-tocopherol both alone and in combination with tamoxifen were tested on MCF-7 and MDA-MB-435 cells. Tamoxifen is the common and mostprescribed drug particularly for estrogen receptor-positive breast cancer. Since it is an estrogen antagonist, it is also known as antiestrogen or SERM (selective estrogen receptor modulator) (National Cancer Institute 2010). Based on the results of this study, tocotrienols in combination with tamoxifen showed antiproliferative effect and synergism in both cell lines. It was suggested that this synergism was the result of different mechanisms for tocotrienols and tamoxifen (Guthrie et al. 1997). In addition, tocotrienols displayed different growth-inhibitory response in MCF-7 and MDA cells. At $8 \mu \mathrm{g} / \mathrm{mL}, 100 \%$ growth inhibition was observed in MCF cells whereas only $44 \%$ inhibition was observed in MDA cells. The $\delta$ - and $\gamma$ - tocotrienols were found to be the most potent growth inhibitors. Since breast cancer patients develop resistance 
to tamoxifen, this may justify the use of tocotrienols and provides a platform for future clinical studies (Nesaretnam et al. 1998).

The antiproliferative and apoptotic effects of different isoforms of tocotrienols in preneoplastic (CL-S1), neoplastic ( $-\mathrm{SA})$, and highly malignant $(+\mathrm{SA})$ mammary epithelial cells have also been studied. The treatment with tocotrienols displayed stronger antiproliferative and apoptotic effects as reported previously (Nesaretnam et al. 1998, 2000, 2007). The inhibitory potency of $\delta$-tocotrienol was higher compared with that of $\gamma$ - and $\alpha$-tocotrienols. Highly malignant $(+\mathrm{SA})$ cells were the most sensitive to the antiproliferative and apoptotic effects of tocotrienols, while the least sensitivity was shown by preneoplastic (CL-S1) cells. It was suggested that the higher biopotency of tocotrienols may be attributed to the presence of unsaturated phytyl chain that enhances the uptake into the cells causing higher concentration in the cells. Eventually, this would lead to greater biological response in reducing the risk of breast cancer (McIntyre et al. 2000).

Apoptosis or programmed cell death involves caspases, which are enzymes responsible for proteolytic cleavages that lead to cell death. Two mechanisms are involved in apoptosis: death receptor (subgroup of tumor necrosis factor (TNF) superfamily) activation, and mitochondrial stress apoptotic signaling pathways. The former involves the activation of caspase- 3 , caspase- 6 , or caspase- 8 while the mitochondrial stress pathway activates caspase-9. Initial studies proposed that tocotrienols mediate antiapoptotic activity through these two mechanisms. In contrast, a study carried out by Shah and Sylvester, on malignant mouse mammary epithelial cells demonstrated that $\gamma$-tocotrienol suppressed PI3 K/PDK/Akt signaling pathway instead of the apoptotic pathways. Upregulation of PI3 K/PDK/Akt mitogenic pathway is a common finding in tumor cells as this pathway increases the expression of FLICE-inhibitory protein (FLIP). FLIP in turn inhibits the activation of caspase- 8 and is associated with increased cell survival and resistance to apoptosis. Therefore, this study suggests that $\gamma$-tocotrienol causes apoptosis by activating caspase- 3 and caspase- 8 independent of the death receptor and mitochondrial apoptotic signaling (Mo and Elson 1999; Yu et al. 1999; Shah and Sylvester 2004).

The involvement of gene expression by tocotrienols has been reported by Nesaretnam et al. Based on cDNA array analysis, tocotrienol treatment in both MCF-7 and MDAMB-231 cells modulated the expression of three genes in a similar manner in both cell lines. Both c-myc-binding protein MM-1 and the 23-kDA highly basic protein (23-kDa HBP) were onefold upregulated. Meanwhile, the interferon inducible protein 9-27 (IFITM-1) showed a twofold downregulation. These proteins may play a significant role in cell cycle and thus exhibit inhibitory effects on cell growth and proliferation. CD74/Ii is involved in presenting processed peptides to the $\mathrm{CD} 4+\mathrm{T}$ lymphocytes. Overexpression of CD 74/Ii facilitates the evasion of tumor cells from the host immune response. Tocotrienols were shown to mediate their antitumor effect by significantly reducing the expression of CD 74/Ii to inhibit the ability of tumor cells to evade from host immune system surveillance (Nesaretnam et al. 2004).

Yap et al., proposed a different mechanism for the effects of tocotrienols in cancer cells. Based on their research using MDA-MB-231 cancer cells, $\gamma$-tocotrienol targeted Id1, an inhibitor of differentiation. Id1 is a member of Helix-Loop-Helix protein family, which plays a major role in breast epithelial cell proliferation, differentiation, and tumorigenesis. Many aggressive and invasive cancers demonstrated upregulation of Id1 gene. Increased Id1 expressions are associated with poor prognosis of cancer. Treatment with $\gamma$-tocotrienol, however, downregulated Id1 by modulating its upstream modulators (Src, Smad 1/5/8, LOX). Phosphorylation of Src and expression of Smad 1/5/8 were suppressed while LOX was inactivated. The invasive nature of cancer cells related to the activation of LOX was also repressed. These were evident from the activation of pro-caspases, fragmentation of DNA, and accumulation of sub-G1 cells (Yap et al. 2010).

Almost $30 \%$ of women diagnosed with early-stage breast cancer have suffered recurrence. Systemic therapies involving cytotoxic and hormonal agents are correlated with the risk of recurrence. Tumors display resistance to anticancer therapy by (a) molecular alteration in the transmembrane drug transport, (b) increase in the repair and detoxification pathways and lastly (c) by molecular alteration of genes resulting in failure in pro-apoptotic response. Currently, the probability for long-term survival in patients may be improved by combining anticancer chemotherapy with tocotrienols (Yap et al. 2010; Yde et al. 2009; Leonessa et al. 1994).

Statins that comprise a class of drugs namely simvastatin, lovastatin, pravastatin, and mevastatin have been commonly used to treat hypercholesterolemia. Recent evidence shows that in addition to lowering biosynthesis of cholesterol in liver, statins also exhibit chemopreventive effects against cancer. This class of drugs competitively inhibits 3-hydroxy-3-methylglutaryl-coenzyme A (HMG$\mathrm{CoA})$ reductase enzyme, due to the structural resemblance with HMGCoA. HMGCoA reductase is the rate limiting enzyme involved in the synthesis of mevalonate which turns on the downstream mitogenic signaling pathway including MAPK and Akt pathways. Unregulated HMGCoA reductase levels were found to be the cause of mevalonate pathway to be constitutively active in cancer cells. Despite being effective in treating cancer, statins are highly associated with dose-related toxicity that even leads to 
death. Individual statins require $2-8 \mu \mathrm{M}$ concentration in blood which is $25 \mathrm{mg} / \mathrm{kg}$ of oral dose to exert their antiproliferative effect in blood. This, however, is not possible due to their detrimental side effects. The $\gamma$-tocotrienol, on the other hand acts by increasing the degradation of HMGCoA reductase enzyme in tumor cells but inadequate absorption in the body limits its use as an anticancer agent. Therefore, $\gamma$-tocotrienol in combination with lower dose of statins potentiates the inhibitory activity of statins. This had a positive outcome whereby a 2- to 11 -fold of marked reduction was observed compared with individual treatment with statin in + SA cells (highly malignant mammary tumor cells) (Wali and Sylvester 2007). In a study by Wali et al., suppression of mevalonate caused statins to display a compensatory upregulation in HMGCA reductase levels. This, however, was not seen in the combined treatment with $\gamma$-tocotrienol. It was suggested that $\gamma$-tocotrienol counteracts this effect in order to prevent the restoration of mevalonate synthesis.

This synergistic combination has another target mechanism, which is the cell cycle arrest. DNA replication and synthesis involve highly regulated phases of G1, S, G2, and $\mathrm{M}$ by cyclin and CDK complexes. Most cancer cells display $50 \%$ of elevated levels of cyclin D1 that is related to a decrease in cell cycle arrest. Cell cycle analysis following combination treatment of statin and tocotrienols displayed cell cycle arrest without causing apoptosis. This is evidence of the effectiveness of this combination treatment as it is cytostatic and not cytotoxic. Therefore, concerns regarding the toxicity factor related to statin intake and bioavailability of $\gamma$-tocotrienol can be avoided (Theriault et al. 1999; Wali et al. 2009a, b, c).

Similar combination treatment has also been tried with celecoxib, a selective COX-2 inhibitor. Over expression of cyclooxygenase-2 (COX-2) has been associated with many types of cancers. Enhanced angiogenesis and metastasis as well as resistance to apoptosis are characteristics of elevated COX-2 levels. Clinical uses of celecoxib have been limited due to cardiovascular and gastrointestinal toxicities (Shafiq et al. 2005). Shirode et al., conducted a study using the combination treatment of subeffective dose of celecoxib and $\gamma$-tocotrienol on + SA tumor cells. This study clearly demonstrates that this combination suppressed COX-2 levels as well as NFkB and Akt mitogenic pathway activation (Shirode and Sylvester 2010).

The fact that the homologs of tocotrienols possess anticancer properties (Sen et al. 2006) had been established by Nesaretnam et al. (1995, 2000). Pierpauli et al., on the other hand, conducted a comparison study between $\alpha-, \gamma$-, and $\delta$-tocotrienols and their synthetic derivative, $\alpha$-tocopheryl succinate ( $\alpha$-TOS). The results revealed that $\gamma$ - and $\delta$-tocotrienols decreased cell viability effectively in Alamar Blue assay at about half of the concentration $\left(\mathrm{IC}_{50}\right)$ needed by $\alpha$-TOS in SKBR3 cancer cell lines. The non-alpha-tocotrienols as well as $\alpha$-TOS induced mitochondrial dysfunction by upregulating the expression of senescent-like growth arrest markers (p53, p21 and p16) (Pierpaoli et al. 2010). Moreover, in order to enhance the therapeutic activity of tocotrienols, esterification of vitamin $\mathrm{E}$ was carried out to yield redox-silent analogues. These esters displayed enhanced stability and were more water-soluble compared with their parent compounds. Additionally, these esters reduced metastasis of MDA-MB-231 cancer cells, thus providing a new perspective in breast cancer research (Behery et al. 2010).

\section{In vivo studies}

A recent study involved inoculation of human breast cancer cells in athymic mice that were then supplemented with TRF. There was a delayed onset in the formation of tumor and a reduction in the tumor size in the treatment group that was only about one-third compared with the control group. This study produced interesting results, as there was a significant 50-fold increase in the tocotrienol level in the adipose tissue compared with mice in the control group. This data confirmed previous study by Okabe et al. (2002), on the accumulation and distribution of tocotrienols in diet. Furthermore, it was suggested that TRF showed antineoplastic activity by mimicking the effects of interferon- $\gamma$ based on the upregulation of certain genes responsible for the antiproliferative effects. On the other hand, the downregulation of c-myc gene that is associated with malignant progression of many human tumors was observed in this study. This study implies that TRF might modulate and enhance the immune response in exerting its antitumor effects (Nesaretnam et al. 2004).

Tocotrienols were recommended as an adjuvant in dendritic cell-based cancer vaccines due to their ability to inhibit the growth of 4T1 murine mammary tumor cells. Tocotrienols also increased the productions of IFN- $\gamma$ and IL-12 to enhance immune response (Abdul Hafid et al. 2010). The intake of $\gamma$-tocotrienols through diet was shown to suppress tumor growth in a syngeneic study (implantation of human breast cancer cells in mice) and triggered apoptosis through endoplasmic reticulum stress, which led to growth arrest and apoptosis in the cancer cells (Healy et al. 2009). Endoplasmic reticulum is a membranous network involved in proper folding and post-translational modification of proteins. Disruption to this network occurs in various forms such as inability to fold mutant proteins, viral infection or diminished energy storage due to hypoxia and low-energy supply. This causes accumulation of unfolded or misfolded proteins known as unfolded protein response (UPR), which subsequently leads to the complete 
shutdown in protein synthesis. Mechanisms to correct this problem will then commence where unfolded proteins will be completely folded whilst misfolded proteins will be subjected to proteasomal degradation. The stress response resulting from the toxic build-up is a normal protective mechanism initiated for cell survival. However, excessive UPR level will lead to an increase in C/EBP homologous protein marker (CHOP). CHOP is a stress marker that switches from pro-survival to pro-apoptotic signal (Healy et al. 2009; Wali et al. 2009d). In a study by Park et al., supplementation with $\gamma$-tocotrienol showed an increase in CHOP. Apart from this, expression of death receptor 5 (DR5) was also upregulated. The DR5 is associated with recruitment of adaptor protein that triggered apoptosis. Additionally, 35\% mammary tumor load was also reduced suggesting the potential of tocotrienol as an anticancer agent targeting endoplasmic reticulum stress (Park and Sanders 2010).

The most recent and novel finding in cancer research is the ability of tocotrienols to increase the expression of interleukin-24 (IL-24) mRNA that suppresses tumor growth and induces apoptosis without harming normal cells. Selvaduray and colleagues reported an increase in the expression of IL-24 mRNA and at the same time IL-8, and vascular endothelial growth factor (VEGF) was downregulated. These compounds play an essential role in tumor growth and development. Reduced tumor incidence and size as well as the potency of the individual tocotrienol compounds in inhibiting 4T1 murine mammary cancer cells were similar to the previous studies (Nesaretnam et al. 2007; McIntyre et al. 2000). These findings confirm the role of IL-24 as an antiangiogenic and antitumor compound (Selvaduray et al. 2010).

\section{Clinical trials}

Researchers from the Malaysian Palm Oil Board have carried out two clinical trials on the efficacy of tocotrienols in breast cancer patients. The first study was conducted to look at the differences in tocopherol and tocotrienol concentrations in malignant and benign adipose tissues in breast cancer patients primarily consuming a palm oil diet. The results revealed a higher concentration of tocotrienols $(\alpha, \delta$ and $\gamma)$, approximately $65 \%$ higher in the adipose tissues of patients with benign breast lumps, compared with that of patients with malignant tumors. Only $\delta$-tocopherol showed a decreased level in malignant adipose tissue whereas $\alpha$ - and $\gamma$-tocopherols did not show any difference. The presence of the unsaturated side chain in tocotrienols allowed for greater penetration into the adipose tissue with better distribution in the saturated fatty layers (Yoshida et al. 2003). The higher concentration of tocotrienols in benign patients may be associated with the protective effect of tocotrienols against breast cancer. The Malaysian diet predominantly contains palm oil with high amounts of tocotrienols, and therefore, it is plausible that dietary tocotrienols in palm oil confer modest protection from breast cancer (Nesaretnam et al. 2007).

The most recent study conducted in MPOB was a novel pilot clinical trial. Previous studies indicated that tocotrienols exert synergistic effects with tamoxifen in vivo. In this clinical trial, which is the first of this nature, women with primary estrogen receptor-positive breast cancer were given a combination of tamoxifen and tocotrienol for 5 years. Interestingly, the risk of dying due to breast cancer decreased by $70 \%$ in patients taking the combination of tocotrienol and tamoxifen compared with the patients only receiving tamoxifen. In the meantime, the risk of recurrence was $20 \%$ lower in those receiving both tocotrienol and tamoxifen compared with the control group only receiving placebo plus tamoxifen. One of the parameters that were studied over the 5 years was the levels of interferon-gamma (IFN- $\gamma$ ) between the two groups of patients. IFN- $\gamma$, which indicates the enhancement of immune response, was found to be significantly elevated in the treatment group compared with the placebo group (Fig. 1). The numbers needed to treat (NNT) is an indication of the effectiveness of the intervention. From this study, the NNT, which was 30 , indicated that the combined treatment of tocotrienol and tamoxifen may prevent one patient in every 30 from dying due to breast cancer (Table 1). Therefore, these results suggest that the synergistic effect of tocotrienols and tamoxifen might contribute to the lower risk of dying and recurrence of breast cancer (Nesaretnam et al. 2010).

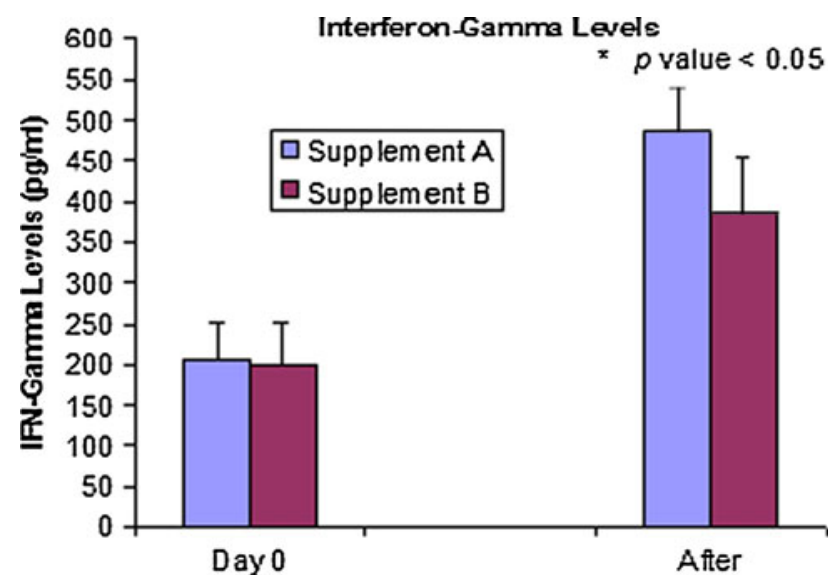

Fig. 1 Comparison of interferon-gamma levels between patients receiving treatment and patients receiving placebo. After completion of year 5, Supplement A TRF + Tamoxifen, Supplement $B$ Placebo + Tamoxifen 
Table 1 Tocotrienol intake and risk of breast cancer-specific death/recurrence in women with early breast cancer and estrogen receptor-positive tumors receiving tamoxifen

\begin{tabular}{|c|c|c|c|c|c|}
\hline \multirow[t]{2}{*}{ Outcome } & \multicolumn{2}{|l|}{ Received tocotrienol } & \multirow{2}{*}{$\begin{array}{l}\text { Relative risk } \\
(95 \% \mathrm{CI})\end{array}$} & \multirow{2}{*}{$\begin{array}{l}\text { Absolute risk } \\
\text { reduction }(95 \% \mathrm{CI})\end{array}$} & \multirow{2}{*}{$\begin{array}{l}\text { Numbers needed } \\
\text { to treat }\end{array}$} \\
\hline & $\begin{array}{l}\text { Yes } \\
n=120 \% \text { (Number) }\end{array}$ & $\begin{array}{l}\text { No } \\
n=120 \% \text { (Number) }\end{array}$ & & & \\
\hline \multicolumn{6}{|l|}{ Primary end point } \\
\hline Breast cancer related death & $0.017(2)$ & $0.050(6)$ & $0.33(0.07-1.62)$ & $-0.03(-0.09$ to 0.02$)$ & 30 \\
\hline \multicolumn{6}{|l|}{ Secondary end point } \\
\hline Recurrence & $0.133(16)$ & $0.167(20)$ & $0.80(0.44-1.47)$ & $-0.03(-0.13$ to 0.06$)$ & 30 \\
\hline
\end{tabular}

\section{Conclusion}

Advances in chemopreventive approaches would be an immense breakthrough in lowering the mortality rate associated with breast cancer in women. Supplementation or treatment with palm tocotrienols has shown encouraging results mainly from in vitro and in vivo studies. The studies conducted demonstrated that tocotrienols have convincing potential in suppressing and inhibiting the growth of mammary tumor cells. Combined treatment with statins, celecoxib, and tamoxifen resulted in a significantly enhanced synergistic response compared with high doses of treatment with individual compounds. Interestingly, this effect was observed using lower doses of the anticancer agent in combination with tocotrienols, suggesting that the toxicity factor related to these drugs may be avoided. The recent clinical trial, even though is reported as a null study, displayed promising reduction in the risk and recurrence free survival in women with early breast cancer. Future clinical studies must be carried out in order to substantiate these findings. Nevertheless, tocotrienols exhibit potential as anticancer agents to be used in combination treatment as well as to enhance therapeutic responsiveness in breast cancer patients.

\section{References}

Abdul Hafid SR, Radhakrishnan AK, Nesaretnam K (2010) Tocotrienols are good adjuvants for developing cancer vaccines. Cancer 10 (Article in press)

Balfe P, McCann A, McGoldrick A, McAllister K, Kennedy M, Dervan P, Kerin MJ (2004) Estrogen receptor $\alpha$ and $\beta$ profiling in human breast cancer. J Cancer Surg 30:469-474

Behery FA, Elnagar AY, Akl MR, Wali VB, Abuasal B, Kaddoumi A, Sylvester PW, El Sayed KA (2010) Redox-silent tocotrienol esters as breast cancer proliferation and migration inhibitors. Bioorg Med Chem 18:8066-8075

Biswas DK, Cruz A, Pettit N, Mutter GL, Pardee AB (2001) A therapeutic target for hormone-independent estrogen receptorpositive breast cancers. Mol Med 7:59-67

Cancer.Org (2010) Cancer Facts and Figures 2010. http:// www.cancer.org/acs/groups/content/@epidemiologysurveilance/ documents/document/acspc-026238.pdf. Accessed 10 Dec 2010
Charts Bin (2010) Current Worldwide Breast Cancer Incidence Rate. http://chartsbin.com/view/yq6. Accessed 8 Dec 2010

Comitato R, Nesaretnam K, Leoni G, Ambra R, Canali R, Bolli A, Marino M, Virgili F (2009) A novel mechanism of natural vitamin $\mathrm{E}$ tocotrienol activity: involvement of $\mathrm{ER} \beta$ signal transduction. Am J Physiol Endocrinol Metab 297:E427-E437

Comitato R, Leoni G, Canali R, Ambra R, Nesaretnam K, Virgili F (2010) Tocotrienols activity in MCF-7 breast cancer cells: involvement of ER $\beta$ signal transduction. Mol Nutr Food Res 54(5):669-678

Cullen R, Maguire TM, McDermott EW, Hill ADK, O'Higgins NJO, Duffy MJ (2001) Studies on oestrogen receptor- $\alpha$ and $-\beta$ mRNA in breast cancer. Eur J Cancer 37:1118-1122

Dayton S, Hashimoto S, Wollman J (1977) Effect of high oleic and high linoleic safflower oils on mammary tumors induced in rats by 7, 12-Dimethylbenzanthracene. J Nutr 107:1353-1360

Fog C, Christensen I, Lykkesgeldt A (2005) Characterization of human breast cancer cell line, MCF-7/RU58R-1, resistant to the pure antiestrogen RU 58, 668. Breast Cancer Res Tr 91:133-144

Guthrie N, Gapor A, Chambers AF, Carroll KK (1997) Inhibition of proliferation of estrogen receptor-negative MDA-MB-435 and positive MCF-7 human breast cancer cells by palm oil tocotrienols and tamoxifen, alone and in combination. J Nutr 127:544S-548S

Healy SJM, Gorman AM, Mousavi-Shafaei P, Gupta S, Samali A (2009) Targetting the endoplasmic reticulum-stress response as an anticancer strategy. Eur J Pharmacol 625:234-246

Ingram DM, Nottager E, Roberts T (1991) The role of diet in the development of breast cancer: a case control study of patients with breast cancer. Br J Cancer 64:187-191

Leonessa F, Jacobson M, Boyle B, Lippman J, McGarvey M, Clarke R (1994) Effect of tamoxifen on the multidrug-resistant phenotype in human breast cancer cells: Isobologram, Drug accumulation, and $\mathrm{Mr}$ 170, 000 glycoprotein (gp170) binding studies. Cancer Res 54:441-447

McIntyre BS, Briski KP, Gapor A, Sylvester PW (2000) Antiproliferative and apoptotic effects of tocopherols and tocotrienols on preneoplastic and neoplastic mouse mammary epithelial cells. Lipids 35:171-180

Mo H, Elson CE (1999) Apoptosis and cell-cycle arrest in human and murine tumors cells are initiated by isoprenoids. J Nutr 129(4):804-813

National Cancer Institute (2010) Surveillance epidemiology and end results. http://seer.cancer.gov/statfacts/html/breast.html. Accessed 8 Dec 2010

National Cancer Institute (2010) Tamoxifen. http://www.cancer.gov/ cancertopics/factsheet/Therapy/tamoxifen. Accessed 8 Dec 2010

Nesaretnam K (2008) Multitargeted therapy of cancer by tocotrienols. Cancer Lett 269:388-395

Nesaretnam K, Guthrie N, Chambers AF, Carroll KK (1992) Effect of tocotrienols on the growth of a human breast cancer cell line in culture. Lipids 30:1139-1145 
Nesaretnam K, Guthrie N, Chambers AF, Caroll KK (1995) Effect of tocotrienols on the growth of a human breast cancer cell line in culture. Lipids 30:1139-1143

Nesaretnam K, Stephen R, Dils R, Darbre P (1998) Tocotrienols inhibit the growth of human breast cancer cells irrespective of estrogen receptor status. Lipids 33:461-469

Nesaretnam K, Dorasamy S, Darbre PD (2000) Tocotrienols inhibit growth of ZR-75-1 breast cancer cells. Int J Food Sci Nutr 51:S95-S103

Nesaretnam K, Ambra R, Selvaduray KR, Radhakrishnan A, Canali R, Virgili F (2004a) Tocotrienol-rich fraction from palm oil and gene expression in human breast cancer cells. Ann N Y Acad Sci 1031:143-157

Nesaretnam K, Ambra R, Selvaduray KR, Radhakrishnan A, Reimann K, Razak G, Virgili F (2004b) Tocotrienol-rich fraction from palm oil affects gene expression in tumors resulting from MCF-7 cell inoculation in athymic mice. Lipids 39:459-467

Nesaretnam K, Yew WW, Wahid MB (2007a) Tocotrienols and cancer: beyond antioxidant activity. Eur J Lipi Sci Technol 109:445-452

Nesaretnam K, Gomez PA, Selvaduray KR, Abdul Razak G (2007b) Tocotrienol levels in adipose tissue of benign and malignant breast lumps in patients in Malaysia. Asia Pac J Clin Nutr 16(3):498-504

Nesaretnam K, Selvaduray KR, Abdul Razak G, Veerasenan SD, Gomez PA (2010) Effectiveness of tocotrienol-rich fraction combine with tamoxifen in the management of women with early breast cancer: a pilot clinical trial. Breast Cancer Res (Article in press)

Okabe M, Oji M, Ikeda I, Tachibana H (2002) Yamada K: tocotrienol level in various tissues of Sprague-Dawley rats after intragastric administration of tocotrienols. Biosci Biotechnol Biochem 66:1768-1771

Breast Cancer.Org (2010) Breast cancer statistics. http://www. breastcancer.org/symptoms/understand_bc/statistics.jsp?gclid= COzU2ZDJ5KUCFUV76wodGCGL0Q. Accessed 8 Dec 2010

Park SK, Sanders BG, Kline K (2010) Tocotrienols induce apoptosis in breast cancer cell lines via an endoplasmic reticulum stressdependant increase in extrinsic death receptor signalling. Breast Cancer Res Treat 124(2):361-375

Pierpaoli E, Viola V, Pilolli F, Piroddi M, Galli F, Provincialli M (2010) $\gamma$ - and $\delta$ - tocotrienols exert a more potent anticancer effect than alpha-tocopheryl succinate on breast cancer cell lines irrespective of HER-2/neu expression. Life Sci 86:668-675

Platet N, Cathiard AM, Gleizes M, Garcia M (2004) Estrogens and their receptors in breast cancer progression: a dual role in cancer proliferation and invasion. Crit Rev Oncol Hematol 51:55-67

Selvaduray KR, Radhakrishnan A, Kutty MK, Nesaretnam K (2010) Palm tocotrienols inhibit proliferation of murine mammary cancer cells and induce expression of interleukin-24 mRNA. J Interferon Cytokine Res 30(12):909-916

Sen CK, Khanna S, Roy S (2006) Tocotrienols: vitamin E beyond tocopherols. Life Sci 78(18):2088-2098
Shafiq N, Malhotra S, Pandhi P, Nada R (2005) Comparative gastrointestinal toxicity of selective cyclooxygenase (COX-2) inhibitors. Indian J Exp Biol 43:614-619

Shah S, Sylvester PW (2004) Tocotrienol-induced caspase-8 activation is unrelated to death receptor apoptotic signaling in neoplastic mammary epithelial cells. Exp Biol Med (Maywood) 229:745-755

Shirode AB, Sylvester PW (2010) Synergistic anticancer effects of combined gamma-tocotrienol and celecoxib treatment are associated with suppression in Akt and NFkB signaling. Biomed Pharmacother 64:327-332

Skliris GP, Leygue E, Watson PH, Murphy LC (2008) Estrogen receptor beta as a therapeutic target. J Steroid Biochem Mol Bio 109:1-10

Sundram K, Khor HT, Ong ASH, Pathmanathan R (1989) Effect of dietary palm oils in mammary carcinogenesis in female rats induced by 7, 12-Dimethylbenz $(\alpha)$ anthracene. Cancer Res 49:1447-1451

Sylvester PW, Shah SJ (2005) Mechanisms mediating the antiproliferative and apoptotic effects of vitamin $\mathrm{E}$ in mammary cancer cells. Front Biosci 10:109-699

Sylvester PW, Russell M, Ip MM, Ip C (1986) Comparative effects of different animal and vegetable fats before and during carcinogenesis administration on mammary tumorigenesis, sexual maturation and endocrine function in rats. Cancer Res 46:757-762

Theriault A, Chao J-T, Wqang Q, Gapor A, Adeli K (1999) Tocotrienol: a review of its therapeutic potential. Clin Biochem 32(5):309-319

Wali VB, Sylvester PW (2007) Synergistic antiproliferative effects of c-tocotrienol and statin treatment on mammary tumor cells. Lipids 42:1113-1123

Wali VB, Bachawal SV, Sylvester PW (2009a) Suppression in Mevalonate synthesis mediates antitumor effects of combined statin and $\gamma$-tocotrienol treatment. Lipids 44:925-934

Wali VB, Bachawal SV, Sylvester PW (2009b) Combine treatment of gamma tocotrienol with statins induce mammary tumor cell cycle arrest in G1. Exp Biol Med (Maywood) 234:639-650

Wali VB, Bachawal SV, Sylvester PW (2009c) Endoplasmic reticulum stress mediates c-tocotrienol and apoptosis in mammary tumor cells. Apoptosis 14:1366-1377

Yap WN, Zaiden N, Tan YL, Ngoh CP, Zhang XW, Wong YC, Yap YL (2010) Id1, inhibitor of differentiation, is a key protein mediating anti-tumor responses of gamma-tocotrienol in breast cancer cells. Cancer Lett 291:187-199

Yde CW, Clausen MP, Bennetzen MV, Lykkesfeldt AE, Mouritsen OG, Guerra B (2009) The antipsychotic drug chlorpromazine enhances the cytotoxic effect of tamoxifen in tamoxifensensitive and tamoxifen-resistant human breast cancer cells. Anti-Cancer Drugs 20(8):723-735

Yoshida Y, Niki E, Noguchi N (2003) Comparative study on the action of tocopherols and tocotrienols as antioxidant: chemical and physical effects. Chem Phys Lipids 123(1):63-75

Yu W, Simmons-Menchaca M, Gapor A, Sanders BG, Kline K (1999) Induction of apoptosis in human breast cancer cells by tocopherols and tocotrienols. Nutr Cancer 33(1):26-32 